\title{
Predictors of mortality and outcomes after retrograde endovascular angioplasty in patients with peripheral artery disease
}

\author{
Pawel Kleczynski ${ }^{1}$, Zoltan Ruzsa ${ }^{2,3}$, Joanna Wojtasik-Bakalarz ${ }^{1}$, Andras Nyerges ${ }^{2}$, Artur Dziewierz ${ }^{1}$, \\ Rafał Januszek ${ }^{4}$, Tomasz Rakowski ${ }^{1}$, Dariusz Dudek ${ }^{1}$, Stanislaw Bartus ${ }^{1}$ \\ ${ }^{1} 2^{\text {nd }}$ Department of Cardiology, Jagiellonian University Medical College, Krakow, Poland \\ ${ }^{2}$ Cardiology Department, Heart and Vascular Center, Semmelweis University, Budapest, Hungary \\ ${ }^{3}$ Invasive Cardiology Department, Bács-Kiskun County Hospital, Teaching Hospital of the Szent-Györgyi Albert Medical University, \\ Kecskemét, Hungary \\ ${ }^{4}$ Department of Clinical Rehabilitation, University of Physical Education, Krakow, Poland
}

Adv Interv Cardiol 2019; 15, 2 (56): 234-239

DOI: https://doi.org/10.5114/aic.2019.81727

\begin{abstract}
A b stract
Introduction: Endovascular revascularization (ER) techniques in patients with peripheral artery disease (PAD) have been developed and became more accessible in recent years. The ER is a first-line treatment in the majority of patients with symptomatic PAD. However, data on assessment of predictors of long-term outcomes of retrograde ER in patients with PAD are scarce.

Aim: To evaluate predictors of long-term outcomes of retrograde ER in patients with chronic total occlusion in lower limb arteries.

Material and methods: We analyzed data of 834 patients who underwent retrograde ER. Baseline clinical characteristics and procedural data were collected. Patients were followed up for 36 months, and the primary endpoint was all-cause mortality.

Results: All patients were symptomatic and had failed antegrade ER. The procedural success rate was $92 \%$. Cumulative all-cause mortality was $13.4 \%$ at 36 -month follow-up. In multivariate analysis history of stroke, Rutherford category, chronic limb ischemia, chronic kidney disease (CKD), chronic obstructive pulmonary disease (COPD) and previous ER of other lesion were independent predictors of a higher mortality rate after 36 months (hazard ratio $(\mathrm{HR})$ for stroke 2.4 , 95\% confidence interval $(\mathrm{Cl}): 1.55-3.66 ; p=$ 0.0002; HR for age per 10 years 1.37, 95\% Cl: 1.15-1.64; $p=0.0002$; HR for Rutherford category 1.63, 95\% Cl: 1.35-1.98; $p<0.0001$, HR for chronic limb ischemia 0.44, 95\% Cl: 0.25-0.8, $p=0.007$; HR for CKD 1.73, 95\% Cl: 1.14-2.56, $p=0.01$; HR for COPD 2.4, $95 \% \mathrm{Cl}: 1.5-3.7, p=0.0004$; HR for previous ER 0.59, $95 \% \mathrm{Cl}: 0.35-0.94, p=0.02$ ).

Conclusions: History of stroke, Rutherford category, chronic limb ischemia, CKD, COPD, and previous ER of other lesion were independently associated with increased risk of all-cause death.
\end{abstract}

Key words: peripheral artery disease, endovascular revascularization, retrograde, mortality, outcomes.

S u m m a ry

The endovascular revascularization (ER) techniques in patients with peripheral artery disease (PAD) have been developed and became more accessible in recent years. However data on assessment of predictors of long-term outcomes of retrograde ER in patients with PAD are scarce. In the present study, we identified predictors of long-term outcomes including mortality in patients with PAD undergoing retrograde ER. Predictors of the composite endpoint (death, reER and amputation) were diabetes, history of stroke and Rutherford grade. Moreover, Rutherford grade, the presence of coronary artery disease and history of stroke were independent predictors of composite end point (death, reER, amputation, myocardial infarction, lower extremity bypass and thrombendaterectomy).

\section{Corresponding author:}

Stanislaw Bartus MD, PhD, $2^{\text {nd }}$ Department of Cardiology, Jagiellonian University Medical College, 17 Mikołaja Kopernika St, 31-501 Krakow, Poland, phone: +48 12 424 7181, e-mail: stanislaw.bartus@uj.edu.pl

Received: 22.10.2018, accepted: 6.01.2019. 


\section{Introduction}

The prevalence of peripheral artery disease (PAD) increases in the aging population and therefore represents a growing problem with diagnostics and management of patients affected by cardiac diseases sharing the same etiology and risk factors [1]. Endovascular revascularization (ER) techniques in patients with PAD have been developed and became more accessible in recent years. The ER is a first-line treatment in the majority of patients with symptomatic PAD. In patients with chronic total occlusion (CTO) of the lower limb arteries and failure of antegrade $E R$, the retrograde access is a treatment option before referral for vascular surgery or conservative treatment. Several studies estimating long-term results of antegrade ER have been published so far, including those comparing antegrade and retrograde access sites $[2,3]$. However, data on assessment of predictors of longterm outcomes of retrograde ER in patients with PAD are scarce.

\section{Aim}

Thus, we aimed to evaluate predictors of long-term outcomes of retrograde ER in patients with CTOs in lower limb arteries.

\section{Material and methods}

\section{Patients}

This study was a prospective, observational registry of 939 consecutive patients in two experienced academic centers, who underwent retrograde recanalization of CTO localized in the iliac artery, superficial femoral artery (SFA), popliteal artery (PA) or below the knee arteries after at least one unsuccessful antegrade attempt. Finally, data of 834 patients with available follow-up were analyzed. Baseline clinical characteristics and procedural data were collected.

\section{Study endpoints}

Patients were followed up for 36 months, and the primary endpoint was all-cause mortality. The secondary composite endpoint consisted of death, re-ER, and amputation. The tertiary composite endpoint consisted of death, re-ER, amputation, stroke, myocardial infarction, arterial bypass, and thromboendarterectomy. The study protocol conforms to the ethical guidelines of the 1975 Declaration of Helsinki with later amendments and was approved by the institutional ethical board. All patients provided informed consent.

\section{Procedural characteristics}

Antegrade failure was defined as an inability to wire the distal part of the vessel after the occlusion via the access site, located in the contralateral artery or proximal to the CTO lesion. According to the local protocol, pa- tients were screened for concomitant diseases, risk factors, and medications. In all patients, the ankle-brachial index (ABI) was determined and severity of PAD was assessed according to the Rutherford, Fontaine and Wagner scales before the procedure. The decision on the use of retrograde recanalization and access site was based on previous angiography. Retrograde recanalization was performed under local anesthesia and required two access sites: antegrade and retrograde. Both proximal and distal punctures were done under the guidance of Doppler ultrasound and/or fluoroscopy. After crossing the occlusion with a wire through the retrograde approach, predilatation with a balloon catheter was done. Stent implantation was left to the operator's decision. After the procedure, the distal sheet was removed immediately and the proximal sheet was maintained up to $4 \mathrm{~h}$. In some cases, due to dissection, the balloon inflations were performed from both ante- and retrograde access (kissing balloons technique) to tear the dissection and facilitate the capture of the wire with a diagnostic catheter.

\section{Pharmacotherapy}

As periprocedural treatment all patients received dual antiplatelet therapy: aspirin $75 \mathrm{mg}$ permanently and clopidogrel $75 \mathrm{mg}$ for 3 months, high dose of statin and according to the local protocol low-molecular-weight heparin for 4 weeks. Additional treatment was accordant to individual risk factors and comorbidities, including beta blockers, statins, and angiotensin-converting-enzyme inhibitors.

\section{Statistical analysis}

Categorical variables are presented as numbers and percentages. Continuous variables are expressed as mean \pm standard deviation or median and interquartile range. Normality was assessed with the Shapiro-Wilk test. Equality of variances was assessed using Levene's test. Multivariable Cox regression analysis was performed to find significant predictors of mortality and outcomes and presented as hazard ratios (HR) with $95 \%$ confidence intervals $(\mathrm{Cl})$. All baseline characteristics and procedural data were tested. Forward selection with a probability value for covariates to enter the model was set at the 0.05 level. All tests were two-tailed, and a $p$-value of $<0.05$ was considered statistically significant. All statistical analyses were performed with JMP, Version 13.1.0 (SAS Institute INC., Cary, NC, USA).

\section{Results}

A total of 939 consecutive patients with PAD requiring revascularization were enrolled between 2009 and 2015 and then followed up. Of them, 834 patients were available for detailed analysis. All patients were symptomatic and had failed antegrade ER. The median age was 68 (60-76) years, and the majority of patients were male 
(62.6\%). Baseline clinical characteristics are presented in Table I. The procedural success rate was $92 \%$. Angiographic and procedural data are shown in Table II. Study endpoints are presented in Figure 1. Cumulative all-cause

Table I. Baseline clinical characteristics $(n=834)$

\begin{tabular}{|c|c|}
\hline Variable & All \\
\hline Age, median (IQR) [years] & $68.0(60.0-76.0)$ \\
\hline Men & $522(62.6 \%)$ \\
\hline Body mass index, median (IQR) $\left[\mathrm{kg} / \mathrm{m}^{2}\right]$ & $27.0(23.71-30.1)$ \\
\hline Arterial hypertension & $735(88.2 \%)$ \\
\hline Diabetes mellitus & $342(49.1 \%)$ \\
\hline Chronic kidney disease & $135(16.2 \%)$ \\
\hline Chronic obstructive pulmonary disease & $98(11.8 \%)$ \\
\hline Coronary artery disease & $339(40.7 \%)$ \\
\hline Hyperlipidemia & $708(84.9 \%)$ \\
\hline History of stroke/transient ischemic attack & $74(8.9 \%)$ \\
\hline Smoking & $463(55.6 \%)$ \\
\hline $\begin{array}{l}\text { Previous endovascular revascularization of } \\
\text { other lesion }\end{array}$ & $183(22.0 \%)$ \\
\hline Previous arterial bypass & $89(10.7 \%)$ \\
\hline $\begin{array}{l}\text { Estimated glomerular filtration rate, median } \\
(\mathrm{IQR})\left[\mathrm{ml} / \mathrm{min} / 1.73 \mathrm{~m}^{2}\right]\end{array}$ & $50.0(40.0-60.0)$ \\
\hline \multicolumn{2}{|l|}{ Rutherford classification: } \\
\hline 0 & 0 \\
\hline 1 & $23(2.8 \%)$ \\
\hline 2 & $131(15.7 \%)$ \\
\hline 3 & $112(13.5 \%)$ \\
\hline 4 & $157(18.9 \%)$ \\
\hline 5 & $134(16.1 \%)$ \\
\hline 6 & $276(33.1 \%)$ \\
\hline \multicolumn{2}{|l|}{ Fontaine scale: } \\
\hline 1 & $1(0.1 \%)$ \\
\hline $2 a$ & $36(4.4 \%)$ \\
\hline $2 b$ & $256(30.7 \%)$ \\
\hline 3 & $149(17.9 \%)$ \\
\hline 4 & $389(46.7 \%)$ \\
\hline 5 & $1(0.1 \%)$ \\
\hline Acute limb ischemia & $42(5 \%)$ \\
\hline Chronic limb ischemia & $468(56.1 \%)$ \\
\hline Intermittent claudication < 50 m & $322(39 \%)$ \\
\hline Ankle brachial index, median (IQR) & $0.6(0.3-0.7)$ \\
\hline
\end{tabular}

Table II. Angiographic and procedural data $(n=834)$

TASC II:

A

$157(18.9 \%)$

B

C $167(20.1 \%)$

D $125(15 \%)$ $383(46 \%)$

Angiography first access site:

Brachial $30(3.6 \%)$

Femoral $599(71.8 \%)$

Radial 204 (24.5\%)

Iliac artery - chronic total occlusion 105 (12.6\%)

Deep femoral artery - significant lesion 42 (5\%)

Common femoral artery:

Chronic total occlusion 33 (4\%)

Calcification:

\begin{tabular}{ll}
\hline Slight & $316(37.9 \%)$ \\
\hline Severe & $131(15.7 \%)$
\end{tabular}

Lesion length [mm] $\quad 14.1 \pm 15.8$

Superficial femoral artery:

Tortuosity:

\begin{tabular}{lc}
\hline Severe & $22(2.7 \%)$ \\
\hline Slight & $285(34.2 \%)$ \\
\hline Chronic total occlusion & $415(49.8 \%)$ \\
\hline
\end{tabular}

Calcification:

\begin{tabular}{lc}
\hline Extreme & $26(3.1 \%)$ \\
\hline Severe & $363(43.6 \%)$ \\
\hline Slight & $248(35.7 \%)$ \\
\hline Lesion length $[\mathrm{mm}]$ & $127.9 \pm 110.3$ \\
\hline
\end{tabular}

Popliteal artery:

\begin{tabular}{ll}
\hline Lesion length $[\mathrm{mm}]$ & $52.3 \pm 48.7$ \\
\hline Chronic total occlusion & $99(11.9 \%)$
\end{tabular}

Tibio-fibular trunk:

Significant lesion $206(24.7 \%)$

Chronic total occlusion $82(9.8 \%)$

Tibialis anterior artery:

\begin{tabular}{lc}
\hline Significant lesion & $387(46.5 \%)$ \\
\hline Chronic total occlusion & $30(3.6 \%)$ \\
\hline Peroneal artery - significant lesion & $305(36.6 \%)$ \\
\hline Tibialis posterior artery: & $391(47.5 \%)$ \\
\hline Significant lesion & $70(8.4 \%)$ \\
\hline Chronic total occlusion & $825.1 \pm 697.4$ \\
\hline Fluoroscopy time [s] & $120.9 \pm 84.5$ \\
\hline Contrast volume [ml] & $3.3 \pm 2.1$ \\
\hline Time from procedure to discharge [days] & $5.5 \pm 5.2$
\end{tabular}


mortality was $13.4 \%$ at 36 months. In multivariate analysis history of stroke, Rutherford category, chronic limb ischemia, chronic kidney disease (CKD), chronic obstructive pulmonary disease (COPD) and previous ER of other lesion were independent predictors of a higher mortality rate after 36 months (HR for stroke 2.4, 95\% confidence interval (Cl): $1.55-3.66 ; p=0.0002 ; \mathrm{HR}$ for age per 10 years $1.37,95 \% \mathrm{Cl}: 1.15-1.64 ; p=0.0002$; HR for Rutherford category $1.63,95 \% \mathrm{Cl}: 1.35-1.98 ; p<0.0001$, HR for chronic limb ischemia 0.44, 95\% Cl: 0.25-0.8, $p=0.007$; HR for CKD 1.73, 95\% Cl: 1.14-2.56, $p=0.01$; HR for COPD 2.4, 95\% Cl: 1.5-3.7, $p=0.0004$; HR for previous ER $0.59,95 \% \mathrm{Cl}: 0.35-0.94, p=0.02)$. Predictors of secondary endpoint (death, reER and amputation) were diabetes $(\mathrm{HR}=1.3,95 \% \mathrm{Cl}: 1.07-1.55, p<0.0075)$, Rutherford category $(\mathrm{HR}=1.27,95 \% \mathrm{Cl}: 1.18-1.37, p<0.0001)$ and history of stroke $(\mathrm{HR}=1.41,95 \% \mathrm{Cl}: 1.05-1.86$, $p=0.02)$, all age-adjusted. Rutherford grade ( $\mathrm{HR}=1.21$, $95 \% \mathrm{Cl}: 1.13-1.3, p<0.0001)$, coronary artery disease $(\mathrm{HR}=1.4,95 \% \mathrm{Cl}: 1.16-1.67, p=0.0003)$ and history of stroke $(\mathrm{HR}=1.38,95 \% \mathrm{Cl}: 1.03-1.81, p=0.02)$ were independent predictors of the tertiary composite end point (death, reER, amputation, myocardial infarction, lower extremity bypass and thromboendarterectomy). Figure 2 presents all-cause mortality during 36 months of follow-up.

\section{Discussion}

In the present study, we identified predictors of longterm outcomes including mortality in patients with PAD undergoing retrograde ER. Predictors of the composite endpoint (death, reER and amputation) were diabetes, history of stroke and Rutherford grade. Moreover, Rutherford grade, the presence of coronary artery disease and history of stroke were independent predictors of composite end point (death, reER, amputation, myocardial infarction, lower extremity bypass and thromboendarterectomy). The ER in PAD treatment has become more accessible and developed in recent years, even in its retrograde technique. Retrograde technique is an interventional option of choice after antegrade attempt failure, especially in high-risk patients who are not suitable candidates for surgical treatment. Importantly, the results of percutaneous angioplasty from the retrograde approach in PAD patients have improved significantly since initial reports [2, 4-7]. Montero-Baker et al. reported that the failure rate of antegrade recanalization can reach up to $20 \%$ in CTO lesions in lower limb arteries [8]. Difficulties in crossing the occlusion are related to the morphology of the plaque as CTOs are harder, more fibrous within the proximal cap, and with diffuse calcification [9-11]. For retrograde puncture, there are a few arteries that can be chosen: the distal segment of the SFA, PA, tibial artery, and pedal artery. The procedure is performed under fluoroscopy or ultrasound guidance to avoid additional bleeding complications and damage of the punctured artery. SFA-CTO prevalence reaches around 50\% in symp-

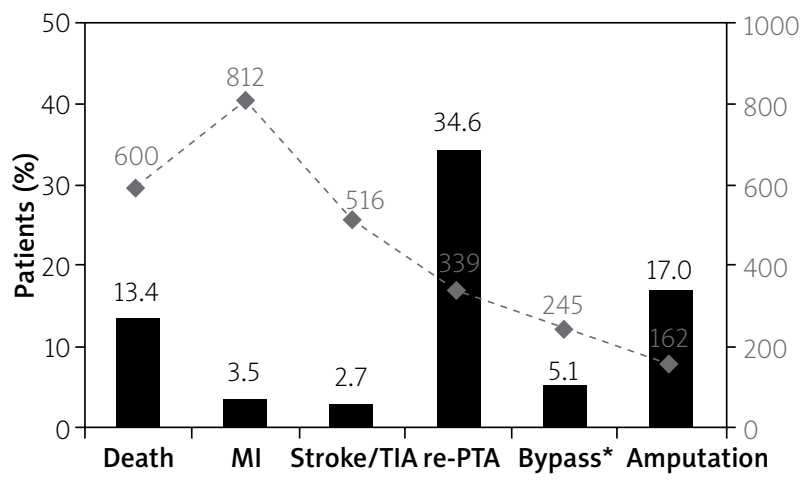

Figure 1. Study endpoints and days-to-event * Lower extremity bypass.

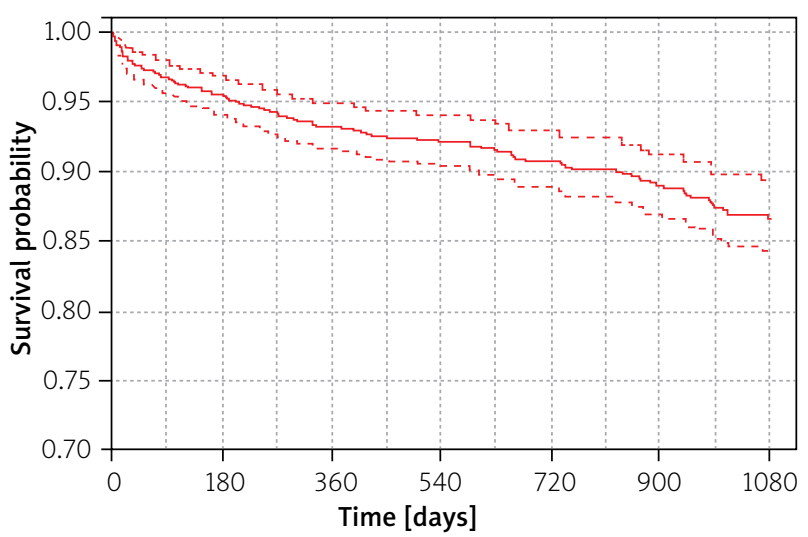

Figure 2. Kaplan-Meier survival curve for patients undergoing retrograde endovascular revascularization at 3-year follow-up

tomatic patients with PAD [12]. Nevertheless, there are still scarce data on large populations that estimate the predictors of long-term treatment results in this group of patients, especially those treated from the retrograde approach. In the current study, the mortality after 36 months of follow-up was $13.4 \%$, which was relatively low compared to previous publications, where mortality in the overall group of patients with PAD treated with endovascular interventions reached $6-50 \%$ with a similar mean age of participants to the current study at baseline and depended on the length of follow-up [13-15]. However, compared to previous studies, the rate of CKD was lower, impacting the mortality. Additionally, the patients were strictly oriented towards drug compliance and administration of the drugs was monitored during every follow-up visit. In another study assessing effectiveness and outcomes after pedal vascular access in critical limb ischemia mortality was $23 \%$ during the mean follow-up of 17 months [16]. A systematic review with more than 1500 patients reported that the 1-year survival rate in patients treated with subintimal recanalization depended on the location of the culprit artery or symptoms before angioplasty and ranged between $65 \%$ and $78 \%$ in patients with critical limb ischemia and crural lesions, and between $86 \%$ and $100 \%$ in those with mixed dis- 
ease and femoral lesions [17]. The study published by Kim et al. reported 1-year mortality following subintimal angioplasty in patients with more advanced and disseminated atherosclerosis (TASC C and D) at 5.5\% [18]. In contrast, in some studies performed in patients with critical or subcritical lower limb ischemia the mortality rate after 5 years of follow-up reached almost 50\% [15]. History of stroke and previous ER of another lesion were also associated with increased mortality risk. However, there are no data available so far regarding this problem. Rutherford category represents the grade of clinical symptoms which are directly affected by the extent of lower limb artery atherosclerosis. The culprit lesion length, clinical state before angioplasty, and the degree of dissemination of atherosclerotic lesions (TASC stage) are among confirmed predictors of clinical outcomes in patients with PAD treated with endovascular interventions [15]. The study published by Murata et al. including 1088 patients, comprising 1306 limbs with critical ischemia treated with endovascular therapy, confirmed the previously discovered relationship in patients with CAD treated with percutaneous intervention [19]. Also, one of several factors for which there is a strong relationship with endovascular outcomes in the follow-up period is CKD. The CKD was found to be a predictor of increased mortality after 4 years of follow-up in patients with PAD treated with $E R$, and it was strictly correlated with the stage of CKD. The projected 4-year mortality in patients with known CKD was $27 \%$, while in patients with other stages it was $46 \%$ (stage 2), 52\% (stage 3), 72\% (stage 4) and $78 \%$ (stage 5) [20]. In a study by Willenberg et al. the presence of CKD was an independent predictor of higher mortality in patients with chronic limb ischemia undergoing ER [21]. Among the most influential mechanisms responsible for worse outcomes after ER of lower limb arteries we can distinguish prothrombotic state related to COPD, elevated proinflammatory markers and markers of oxidative stress $[22,23]$. The relationship between COPD and future cardiovascular adverse events in patients with CAD has repeatedly been demonstrated so far [24, 25]. Diabetes mellitus is a well-established risk factor for poor outcomes in cardiovascular diseases. In a study by Neupane et al. with 714 patients, diabetes mellitus was independently associated with higher mortality, major amputations and repeat revascularization at 5 years [26].

\section{Study limitations}

Only patients with available long-term follow-up were enrolled in the current analysis. Due to drop-offs, the baseline risk characteristics and mortality rates might be underestimated.

\section{Conclusions}

History of stroke, Rutherford category, chronic limb ischemia, CKD, COPD, and previous ER of other lesion were independently associated with increased risk of long-term all-cause death.

\section{Conflict of interest}

The authors declare no conflict of interest.

\section{References}

1. Aboyans V, Ricco JB, Bartelink MEL, et al. 2017 ESC Guidelines on the Diagnosis and Treatment of Peripheral Arterial Diseases, in collaboration with the European Society for Vascular Surgery (ESVS). Eur J Vasc Endovasc Surg 2018; 55: 305-68.

2. Ruzsa Z, Wojtasik-Bakalarz J, Nyerges A, et al. Long-term follow-up after retrograde recanalization of superficial femoral artery chronic total occlusion. J Invasive Cardiol 2017; 29: 336-9.

3. Evans C, Peter N, Gibson M, et al. Five-year retrograde transpopliteal angioplasty results compared with antegrade angioplasty. Ann R Coll Surg Engl 2010; 92: 347-52.

4. Tønnesen KH, Sager P, Karle A, et al. Percutaneous transluminal angioplasty of the superficial femoral artery by retrograde catheterization via the popliteal artery. Cardiovasc Intervent Radiol 1988; 11: 127-31.

5. Zaitoun R, lyer SS, Lewin RF, et al. Percutaneous popliteal approach for angioplasty of superficial femoral artery occlusions. Cathet Cardiovasc Diagn 1990; 21: 154-8.

6. Heenan SD, Vinnicombe SJ, Buckenham TM, et al. Percutaneous transluminal angioplasty by a retrograde subintimal transpopliteal approach. Clin Radiol 1994; 49: 824-7.

7. Yilmaz S, Sindel T, Ceken K, et al. Subintimal recanalization of long superficial femoral artery occlusions through the retrograde popliteal approach. Cardiovasc Intervent Radiol 2001; 24: 154-60.

8. Montero-Baker M, Schmidt A, Bräunlich S, et al. Retrograde approach for complex popliteal and tibioperoneal occlusions. J Endovasc Ther 2008; 15: 594-604.

9. Chin H'ng MW, Punamiya S. An innovative modification of the retrograde approach to angioplasty and recanalization of the superficial femoral artery. Diagn Interv Radiol 2014; 20: 164-7.

10. Fanelli F, Lucatelli P, Allegritti M, et al. Retrograde popliteal access in the supine patient for recanalization of the superficial femoral artery: initial results. J Endovasc Ther 2011; 18: 503-9.

11. Lee HJ, Park SW, Chang S, et al. Strategies for successful percutaneous revascularization of chronic total occlusion of the femoropopliteal arteries when the antegrade passage of a guide wire fails. Korean J Radiol 2012; 13: 467-75.

12. Nadal LL, Cynamon J, Lipsitz EC, et al. Subintimal angioplasty for chronic arterial occlusions. Tech Vasc Interv Radiol 2004; 7: 16-22.

13. Wojtasik-Bakalarz J, Arif S, Chyrchel M, et al. Twelve months follow-up after retrograde recanalization of superficial femoral artery chronic total occlusion. Adv Interv Cardiol 2017; 13: 47-52.

14. Bertelè V, Roncaglioni MC, Pangrazzi J, et al. Clinical outcome and its predictors in 1560 patients with critical leg ischaemia. Chronic Critical Leg Ischaemia Group. Eur I Vasc Endovasc Surg 1999; 18: 401-10.

15. Löfberg AM, Karacagil S, Ljungman C, et al. Percutaneous transluminal angioplasty of the femoropopliteal arteries in limbs with chronic critical lower limb ischemia. J Vasc Surg 2001; 34: 114-21.

16. Bazan HA, Le L, Donovan M, et al. Retrograde pedal access for patients with critical limb ischemia. J Vasc Surg 2014; 60: 375-81. 
17. Met R, Van Lienden KP, Koelemay MJ, et al. Subintimal angioplasty for peripheral arterial occlusive disease: a systematic review. Cardiovasc Intervent Radiol 2008; 31: 687-97.

18. Kim SJ, Kim W, Kim JB, et al. Determinants of procedural success and patency following subintimal angioplasty in patients with TASC C and D femoropopliteal arterial disease. Circ J 2010; 74: 1959-64.

19. Murata N, Soga Y, lida O, et al. Complex relationship of body mass index with mortality in patients with critical limb ischemia undergoing endovascular treatment. Eur J Vasc Endovasc Surg 2015; 49: 297-305.

20. Lüders F, Bunzemeier H, Engelbertz C, et al. CKD and acute and long-term outcome of patients with peripheral artery disease and critical limb ischemia. Clin J Am Soc Nephrol 2016; 11: 216-22.

21. Willenberg T, Baumann F, Eisenberger U, et al. Impact of renal insufficiency on clinical outcomes in patients with critical limb ischemia undergoing endovascular revascularization. J Vasc Surg 2011; 53: 1589-97.

22. Mejza F, Lamprecht B, Niżankowska-Mogilnicka E, et al. Arterial and venous thromboembolism in chronic obstructive pulmonary disease: from pathogenic mechanisms to prevention and treatment. Pneumonol Alergol Pol 2015; 83: 485-94.

23. Maclay JD, McAllister DA, Johnston S, et al. Increased platelet activation in patients with stable and acute exacerbation of COPD. Thorax 2011; 66: 769-74.

24. Christie DJ, Kottke-Marchant K, Gorman RT. Hypersensitivity of platelets to adenosine diphosphate in patients with stable cardiovascular disease predicts major adverse events despite antiplatelet therapy. Platelets 2008; 19: 104-10.

25. Gianetti J, Parri MS, Sbrana S, et al. Platelet activation predicts recurrent ischemic events after percutaneous coronary angioplasty: a 6 months prospective study. Thromb Res 2006; 118 : 487-93.

26. Neupane S, Edla S, Maidona E, et al. Long-term outcomes of patients with diabetes mellitus undergoing percutaneous in tervention for popliteal and infrapopliteal peripheral arterial disease. Catheter Cardiovasc Interv 2018 Mar 14. doi: 10.1002/ ccd. 27571 